\title{
Strong Radiation-Damping Effects in a Gamma-Ray Source Generated by the Interaction of a High-Intensity Laser with a Wakefield-Accelerated Electron Beam
}

\author{
A. G. R. Thomas, ${ }^{1,2}$ C. P. Ridgers, ${ }^{3}$ S. S. Bulanov, ${ }^{4}$ B. J. Griffin, ${ }^{2}$ and S. P. D. Mangles ${ }^{5}$ \\ ${ }^{1}$ Centre for Ultrafast Optical Science, University of Michigan, Ann Arbor, Michigan 48109, USA \\ ${ }^{2}$ Department of Nuclear Engineering and Radiological Sciences, University of Michigan, Ann Arbor, Michigan 48109, USA \\ ${ }^{3}$ Clarendon Laboratory, University of Oxford, Parks Road, Oxford OX1 3PU, United Kingdom \\ ${ }^{4}$ University of California at Berkeley, Berkeley, California 94720, USA \\ ${ }^{5}$ Blackett Laboratory, Imperial College London, London SW7 2AZ, United Kingdom \\ (Received 1 April 2012; revised manuscript received 7 July 2012; published 19 October 2012)
}

\begin{abstract}
A number of theoretical calculations have studied the effect of radiation-reaction forces on radiation distributions in strong-field counterpropagating electron-beam-laser interactions, but could these effects-including quantum corrections-be observed in interactions with realistic bunches and focusing fields, as is hoped in a number of soon-to-be-proposed experiments? We present numerical calculations of the angularly resolved radiation spectrum from an electron bunch with parameters similar to those produced in laser-wakefield-acceleration experiments, interacting with an intense, ultrashort laser pulse. For our parameters, the effect of radiation damping on the angular distribution and energy distribution of photons is not easily discernible for a realistic moderate-emittance electron beam. However, experiments using such a counterpropagating beam-laser geometry should be able to measure these effects using current laser systems through measurement of the electron-beam properties. In addition, the brilliance of this source is very high, with peak spectral brilliance exceeding $10^{29}$ photons s${ }^{-1} \mathrm{~mm}^{-2} \mathrm{mrad}^{-2}(0.1 \% \text { bandwidth })^{-1}$ with an approximately $2 \%$ conversion efficiency and with a peak energy of $10 \mathrm{MeV}$.
\end{abstract}

DOI: 10.1103/PhysRevX.2.041004

\section{INTRODUCTION}

The recent development of ultrahigh-intensity laser systems has generated a great amount of interest in a class of well-known theoretical problems involving the interaction of strong fields with relativistic electron beams that have not been experimentally demonstrated. Relativistic electron beams are regularly measured in experiments by laser-wakefield acceleration (LWFA) [1-4] and are characterized by being of relatively high current density in short bunches. In laser-wakefield acceleration, oscillations of the electrons in the electromagnetic fields of electron plasma cavities created by laser-driven ponderomotive expulsion have been shown to result in extremely bright sources of $x$ rays [5-11].

Another proposed source of radiation using the wakefield-accelerated electron beam is Thomson or Compton backscattering from a second laser [12-21]. This source has been recently demonstrated in the gamma-ray regime using a single laser pulse by reflection from a foil [22]. In this scheme, a counterpropagating laser is used as a short wavelength undulator for producing highbrightness, monochromatic gamma rays. An undulator in a conventional synchrotron is characterized by a strength

Published by the American Physical Society under the terms of the Creative Commons Attribution 3.0 License. Further distribution of this work must maintain attribution to the author(s) and the published article's title, journal citation, and DOI.
Subject Areas: Computational Physics, Optics, Plasma Physics

parameter $K$ that characterizes the oscillation amplitude relative to wavelength. For small $K$, the radiation is monochromatic. For large $K$, the radiation is characterized by a synchrotronlike spectrum [23]. In the counterpropagating laser scheme, the field-strength parameter (normalized peak vector potential) $a_{0}=\left|e F_{0}\right| / m_{e} c \omega_{0}$ is analogous to $K . F_{0}$ is the peak electric field strength of a laser with central angular frequency $\omega_{0}$. For a laser with $a_{0} \ll 1$ $\left(I \lambda^{2} \ll 10^{18} \mathrm{~W} \mathrm{~cm}^{-2}\right)$, the radiation is monochromatic. For $a_{0}>1$, harmonics in the radiation spectrum start to appear, and for $a_{0} \gg 1$, the spectrum becomes broad. For linear polarization of the laser, there is also longitudinal motion due to the Lorentz force; therefore, downshifting of the fundamental frequency occurs $[24,25]$. The monochromatic regime using a relativistic electron bunch has been proposed as a good source for applications [24,26-28]. In addition, experiments using this counterpropagating geometry with a very high-intensity laser (Fig. 1) should be an interesting test bed for studying radiation-reaction forces and nonlinear quantum electrodynamics [29], due to the high field strength in the electron rest frame.

The transverse component of the laser vector potential is Lorentz invariant, so the radiation emission of an $a_{0} \leq 1$ interaction is very different from an $a_{0} \gg 1$ interaction independent of the reference frame (and therefore of electron energy, in the colliding geometry). The emission of photons in such processes clearly indicates that a force should be applied to the electron to conserve momentum. Conversely, the electric field strength is not a Lorentz 


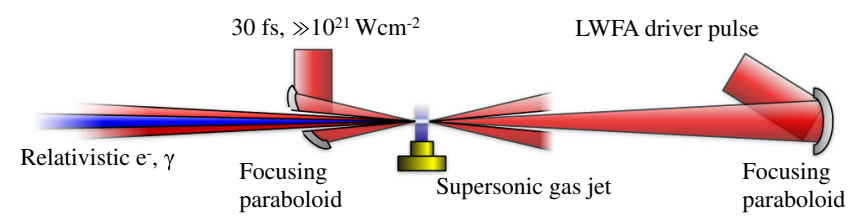

FIG. 1. Schematic drawing of counterpropagating laserbeam-interaction geometry using laser-wakefield-accelerated electrons.

invariant; hence, the electron energy in this geometry may be crucial to determining whether the field is quantum electrodynamically strong or not.

In this paper, radiation-damping effects on the full angular and energy distribution of photons produced in the counterpropagating geometry interaction between a tightly focused ultrashort pulse with intensity of order $10^{22} \mathrm{~W} \mathrm{~cm}^{-2}$ and an electron beam are studied by solving modified classical equations of motion numerically and generating spectra with a numerical radiation spectrometer [30]. The layout of the manuscript is as follows: First, we parametrize the interplay between the field strength $a_{0}$ and electron energy $\gamma m_{e} c^{2}$ in the colliding pulse geometry, and identify the regime relevant to near-term experiments where radiation damping is strong but quantum electrodynamic effects are relatively small. Next, we introduce the numerical model for calculating both the electron dynamics and the radiation spectra. We then proceed to calculate the $\gamma$-ray spectrum with realistic conditions and then examine the effect of radiation reaction on the photon and electron phase-spaces. Finally, we show that semiclassical corrections to the radiation-reaction force may be observable in experiments.

\section{PARAMETRIZING STRONG FIELD INTERACTIONS}

\section{A. Radiation-reaction-force effects}

Although radiation force is properly described by quantum electrodynamics, there exists a classical form for a radiation force that is self-consistent within the limit that the acceleration time scale is much larger than $\tau_{0}=$ $2 e^{2} / 3 m c^{3}=6.4 \times 10^{-24} \mathrm{~s}[31,32]$. In the beam-laser geometry described here, this condition corresponds to $\gamma^{2} a_{0} \omega_{0} \tau_{0} \ll 1$. The effect of this force is principally a damping of motion due to loss of the momentum to the radiation. The Lorentz-Abraham-Dirac equation is a thirdorder differential equation of motion for a charged particle in the presence of accelerating forces; it includes the change of momentum due to the radiation generated by the charge. The force on an electron is given in covariant form by

$$
\frac{d}{d \tau} v^{\mu}=-\frac{e}{m_{e}}\left(F^{\mu \nu} v_{\nu}+\tau_{0} D^{\mu}\right)
$$

where $D^{\mu}$ is the radiation-reaction (damping) force, $F^{\mu \nu}=$ $\partial^{\mu} A^{\nu}-\partial^{\nu} A^{\mu}$ is the electromagnetic field tensor, and $v_{\nu}=d x^{\nu} / d \tau=\{\gamma c,-\gamma \mathbf{v}\}$ is the particle four-velocity. For an $800 \mathrm{~nm}$ laser interacting with a $200 \mathrm{MeV}$ beam, the validity condition above is reasonably fulfilled only for $a_{0} \lesssim 50$ (i.e., where the acceleration time is of the order $10 \tau_{0}$ ). It is worth emphasizing that using this model outside of this limit may not be accurate.

The radiation-reaction force, according to the LorentzAbraham-Dirac model, is a source of much controversy precisely because it is a third-order differential equation, which allows, for example, for self-accelerating solutions that do not conserve energy. Various authors have reformulated the equation to eliminate the third-order term. (See Sokolov [33], Hammond [34], and references within.) These are generally identical to first order in $\tau_{0}$ (and are therefore basically all equivalent to the LandauLifshitz form of the radiation-reaction force [35]), but are otherwise not identical. The modified force can be written in the form [32]

$$
\frac{d}{d \tau} v^{\mu}=-\frac{e}{m_{e}}\left[F^{\mu \nu} v_{\nu}-\tau_{0} P^{\mu \alpha} \frac{d}{d \tau}\left(F_{\alpha}^{\nu} v_{\nu}\right)\right]
$$

where $P^{\mu \alpha}=\eta^{\mu \alpha}+v^{\mu} v^{\alpha} / c^{2}$ and $\eta^{\mu \nu}$ is the Minkowski metric tensor with trace -2 . In Ref. [36], several examples show that the solutions of the Lorentz-Abraham-Dirac model and Eq. (2) are identical in the classical regime.

One of the interesting phenomena arising from this laser-electron interaction is that the radiation damping is theoretically predicted to be so extreme that, for a sufficiently intense laser, the electron beam may lose almost all its energy in the interaction time. In particular, Koga et al. have studied the effect of radiation damping on the radiation spectrum [20]. Di Piazza et al. also studied the effect of radiation damping on the angular distribution of radiation [37]. The effects of real-world conditions on the radiation spectrum emitted have also been studied previously, for example, the effects of higher-order field corrections for tightly focused pulses $[38,39]$.

Radiation damping can be parametrized by considering the energy loss of the electron due to the most significant damping term [20,29]. Here we proceed from Eq. (2), where, ignoring terms of $\tau_{0}^{2}$ and higher and the Schott term, the damping contribution can be written in the form [32]

$$
\frac{d}{d \tau} v^{\mu}=-\frac{e}{m_{e}} F_{\alpha}^{\nu} v_{\nu}\left[\eta^{\alpha \mu}-\tau_{0} \frac{e}{m_{e} c^{2}} v_{\beta} v^{\mu} F^{\alpha \beta}\right] .
$$

The electromagnetic four-force can be written in the form

$$
F^{\alpha \nu} v_{\nu}=-\frac{d A^{\alpha}}{d \tau}+v_{\nu} \partial^{\alpha} A^{\nu}
$$

For the case of a linearly polarized plane wave, $A^{\mu}=$ $\Re\left[\left\{A_{0}\right\}^{\mu} e^{i \kappa_{\alpha} x^{\alpha}} f\left(\kappa_{\beta} x^{\beta} / \omega_{0} t_{\mathrm{L}}\right)\right]$, where $\kappa_{\alpha}$ is the four-wave vector $\kappa_{\alpha}=\omega_{0}\{1,-\hat{\mathbf{k}} / c\}, f\left(\kappa_{\alpha} x^{\alpha} / \omega_{0} t_{\mathrm{L}}\right)$ is a function describing the temporal envelope, and $t_{\mathrm{L}}$ is the pulse 
duration, interacting with a counterpropagating electron with initial Lorentz factor $\gamma_{0}$ obeying $a_{0} \ll \gamma_{0} \ll$ $\left(a_{0} \omega_{0} \tau_{0}\right)^{-1 / 2}$, and the zeroth component is well approximated by

$$
\frac{d \gamma}{d \tau}=-\gamma \tau_{0} \frac{d a^{\mu}}{d \tau} \frac{d a_{\mu}}{d \tau},
$$

where $a^{\mu}=e A^{\mu} / m_{e} c$. The condition on $\gamma$ is set so that the longitudinal Lorentz force is minimized but radiation damping does not affect the transverse oscillations of the electron. For a slowly varying Gaussian envelope, i.e., $(1 / f) d f / d \tau \ll \kappa_{\mu} v^{\mu}$ with $f=\exp \left[-\left(\kappa_{\alpha} x^{\alpha} / \omega_{0} t_{L}\right)^{2}\right]$, and averaging over the fast oscillations, we can integrate to obtain the total energy loss by the particle:

$$
\frac{\Delta \gamma_{\infty}}{\gamma_{0}}=\frac{\sqrt{\frac{\pi}{2}} \tau_{0} t_{\mathrm{L}} \omega_{0}^{2} \gamma_{0} a_{0}^{2}}{1+\sqrt{\frac{\pi}{2}} \tau_{0} t_{\mathrm{L}} \omega_{0}^{2} \gamma_{0} a_{0}^{2}} .
$$

This is similar to the result in Ref. [36], but with a different definition for the pulse duration because here $t_{\mathrm{L}}$ is close to the full-width-at-half-maximum duration commonly used in experiments. From this expression, we can define a parameter $\psi=10 \sqrt{\frac{\pi}{2}} \tau_{0} t_{\mathrm{L}} \omega_{0}^{2} \gamma_{0} a_{0}^{2} t_{\text {rad }}$, for a particular characteristic time scale for radiation damping $t_{\text {rad }}$, such that

$$
\frac{\Delta \gamma_{\infty}}{\gamma_{0}}=\frac{0.1 \psi\left(t / t_{\mathrm{rad}}\right)}{1+0.1 \psi\left(t / t_{\mathrm{rad}}\right)},
$$

which clearly defines strong radiation damping for $\psi \geq 1$ and weak radiation damping for $\psi \ll 1$. Here we choose $t_{\mathrm{rad}}=2 \pi / \omega_{0}$ - that is, a laser period-which is slightly different from the choice of Koga et al. [20], who chose the pulse duration for $t_{\text {rad }}$. However, we have also added a factor of 10 into $\psi$ such that $\psi=1$ corresponds to a $10 \%$ energy loss in a single cycle, which therefore results in a condition similar to that of Koga et al. [20], since they considered an approximately 10 -cycle pulse. In addition, a $10 \%$ loss in a single cycle can reasonably be defined as the threshold of "significant" damping. Hence,

$$
\psi=10 \sqrt{2 \pi^{3}} \omega_{0} \tau_{0} \gamma_{0} a_{0}^{2} .
$$

For an 800-nm laser, $\psi=1.2 \times 10^{-6} \gamma_{0} a_{0}^{2}$. The condition $\psi=1$ leads to the condition for the laser-pulse vector potential, i.e., the strong radiation-damping regime is realized for

$$
a_{0}>a_{\mathrm{rad}}=\left(10 \sqrt{2 \pi^{3}} \omega_{0} \tau_{0} \gamma_{0}\right)^{-1 / 2}
$$

\section{B. Quantum electrodynamics effects}

Quantum electrodynamically strong interactions are parametrized by relativistically and gauge-invariant parameters $\chi_{e}=\left\|F_{\mu \nu} v^{\nu}\right\| /\left(c E_{\mathrm{cr}}\right)$ and $\chi_{\gamma}=\left\|F_{\mu \nu} \hbar k^{\nu}\right\| /$ $\left(m_{e} c E_{\mathrm{cr}}\right)$ [40], where $\hbar k^{\nu}$ is the four-momentum of a photon and $E_{\mathrm{cr}}=m_{e}^{2} c^{3} / e \hbar=1.32 \times 10^{18} \mathrm{Vm}^{-1}$ is the critical field of quantum electrodynamics. These parameters determine the rates of photon creation by an electron or of an electron-positron pair creation by a high-energy photon in a strong electromagnetic field, the latter being the Breit-Wheeler process [41]. The photon-emission probability for $\chi_{e} \ll 1$ is approximately $\left(5 \alpha m_{e}^{2} / 2 \sqrt{3} p_{0}\right) \chi_{e}$ and for $\chi_{e} \gg 1$ is approximately $\left[14 \Gamma(2 / 3) \alpha m_{e}^{2} / 27 p_{0}\right]\left(3 \chi_{e}\right)^{2 / 3}$, where $p_{0}$ is the electron energy and $\Gamma(z)=\int_{0}^{\infty} t^{z-1} e^{-t} d t$ is the Euler gamma function, and $\alpha=e^{2} / 4 \pi \epsilon_{0} \hbar c=1 / 137$ is the fine-structure constant [40]. The pair-production probability by a photon for $\chi_{\gamma} \ll 1$ is approximately $\left(3 \sqrt{3} \alpha m_{e}^{2} / 16 \sqrt{2} k_{0}\right) \chi_{\gamma} \times$ $\exp \left(-8 / 3 \chi_{\gamma}\right)$ and for $\chi_{\gamma} \gg 1$ is approximately $\left[15 \Gamma^{4}(2 / 3) \alpha m_{e}^{2} / 28 \pi k_{0}\right]\left(3 \chi_{\gamma}\right)^{2 / 3}$, where $k_{0}$ is the photon energy [40]. It has previously been shown that extremely high intensity counterpropagating laser pulses could lead to prolific pair production [42-44].

For multi-100 TW lasers, such as the Hercules [45] or Astra Gemini [46] lasers, with focused field strength $|E| \sim 10^{-3} E_{\mathrm{cr}}$, interaction with $\mathrm{GeV}$-energy electron beams should be sufficient to achieve $\chi_{e} \sim 1$ [47-49]. However, the conversion of emitted photons into electron-positron pairs is suppressed due to the $\exp \left(-8 / 3 \chi_{\gamma}\right)$ in the expression for the probability for $\chi_{\gamma} \ll 1$.

A notable experiment in a similar geometry, using the 46-GeV electron beam from the Stanford Linear Accelerator (SLAC) colliding with a laser with intensity of $I_{0} \sim 10^{18} \mathrm{~W} \mathrm{~cm}^{-2}$, was an important demonstration of nonlinear quantum electrodynamics (multiphoton BreitWheeler pair production) [50]. A simplified version of the parameter $\chi_{e}$ for the situation of an electron beam with energy $E=\gamma_{0} m_{e} c^{2}$ colliding with a laser field with field-strength parameter $a_{0}$ can be written as [49]

$$
\chi_{e}=\frac{2 \hbar}{m_{e} c^{2}} \omega_{0} \gamma_{0} a_{0}
$$

For an 800-nm laser system, Eq. (10) gives $\chi_{e}=$ $6 \times 10^{-6} \gamma_{0} a_{0}$. For the SLAC experiment (using a 527-nm laser), the small $a_{0}\left(a_{0}<1\right)$ is compensated for by the high beam energy $\left(\gamma_{0} \sim 10^{5}\right)$, so that $\chi_{e} \approx 0.4$.

Quantum-electrodynamics effects may be considered significant when the energy of the emitted photons becomes of the order of the electron energy, $\hbar \omega \gtrsim \gamma_{0} m_{e} c^{2}$. For a head-on collision of an electron and a laser pulse, a characteristic emitted photon energy is $\hbar \omega \approx \hbar \omega_{0} a_{0} \gamma_{0}^{2}$ [29], which corresponds to the condition $\chi_{e} \sim 1$. Hence, quantum-electrodynamics effects may be considered to be strong for a field strength of

$$
a_{0}>a_{Q}=\frac{m_{e} c^{2}}{2 \hbar \omega_{0} \gamma_{0}} .
$$

However, quantum effects in the radiation damping of electrons becomes noticeable for much lower laser field 
strengths. It is well known $[40,49,51,52]$ that the classical description of an electron radiating in a strong electromagnetic field overestimates the total emitted power. This understanding is connected to the fact that, in the quantum description, the emitted photon energy may not exceed the electron energy, whereas the classical approach does not have such a restriction. This effect can be approximately taken into account by introducing a function $g\left(\chi_{e}\right)$ into the expression for the total power of emitted radiation [49,51,52]. $g\left(\chi_{e}\right)$ enters the equation of motion by modifying the expression for the radiation-reaction force as

$$
\frac{d}{d \tau} v^{\mu}=-\frac{e}{m_{e}} F_{\alpha}^{\nu} v_{\nu}\left[\eta^{\alpha \mu}-g\left(\chi_{e}\right) \tau_{0} \frac{e}{m_{e} c^{2}} v_{\beta} v^{\mu} F^{\alpha \beta}\right]
$$

The strong damping parameter $\psi$ can be modified to include this quantum effect to obtain a parameter $\psi_{Q}=$ $\left\langle g\left(\chi_{e}\right)\right\rangle \psi$, where $\left\langle g\left(\chi_{e}\right)\right\rangle$ is the time average of the $g$ factor. To make this modification, we use a polynomial fraction fit to data for $g\left(\chi_{e}\right)$ given in Ref. [52],

$$
g\left(\chi_{e}\right)=\left(3.7 \chi_{e}^{3}+31 \chi_{e}^{2}+12 \chi_{e}+1\right)^{-4 / 9},
$$

which, for $\chi_{e} \rightarrow 0, g \rightarrow 1$. The condition $\chi_{e}=1$ corresponds to $g\left(\chi_{e}\right)=0.18$, but, even for $\chi_{e}=0.1$, this factor has a value of $g\left(\chi_{e}\right)=0.66$. The time-averaged field-strength parameter $a_{0} / \sqrt{2}$ (for linear polarization) is used to approximate $\left\langle g\left(\chi_{e}\right)\right\rangle \approx g\left(\left\langle\chi_{e}\right\rangle\right)$, which is valid for $\chi_{e} \ll 1$.

The modified strong damping parameter $\psi_{Q}$ for an 800-nm-wavelength laser is shown in Fig. 2 as a function of $a_{0}$ and $\gamma_{0}$. As described in Refs. [49,52], for $\chi_{e} \sim 0.1$, the spectrum emitted should not change significantly in shape, but $\left\langle g\left(\chi_{e}\right)\right\rangle \approx g\left(\left\langle\chi_{e}\right\rangle\right)$ indicates that the energy loss

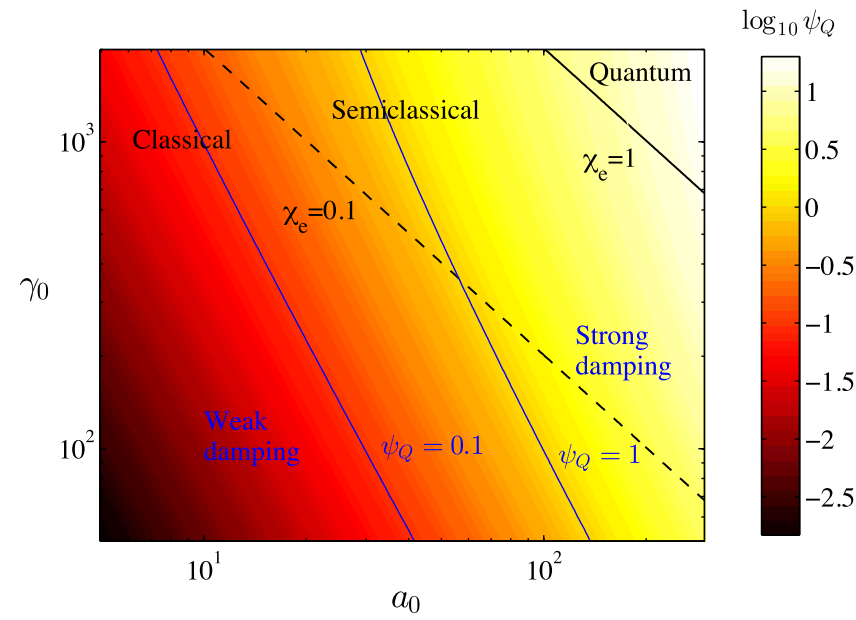

FIG. 2. The function $\psi_{Q}$ as a function of $a_{0}$ and $\gamma_{0}$ for an 800-nm central-wavelength laser. The solid line indicates the threshold between classical and quantum radiation-reaction forces, and the dashed line indicates the threshold at which $g\left(\chi_{e}\right)$ begins to be significant. of the electron beam due to radiation damping should change by a measurable amount. This finding is also consistent with what we observe with our model.

\section{Parameter regimes involving $\chi_{e}$ and $\psi_{Q}$}

The counterpropagating-geometry laser-electron beam experiment is an excellent test bed for studying quantum electrodynamics and strong radiation-damping effects because we can choose between strongly radiation-damped behavior $\left(\psi_{Q} \geqslant 1\right)$ or fields that are quantum-electrodynamically strong $\left(\chi_{e} \gtrsim 1\right)$, and a situation in which both $\psi_{Q} \gtrsim 1$ and $\chi_{e} \gtrsim 1$ simultaneouslyconditions where even more exotic effects may occur. These effects are controlled through variation of the laser-field strength $a_{0}$, central frequency $\omega_{0}$, and the electron-beam energy $\gamma_{0} m_{e} c^{2}$. We can compare the requirements for $a_{0}, \omega_{0}$, and $\gamma_{0}$ for the interaction to be in the strong radiation-damping regime or the quantumelectrodynamics-dominant regime in experiments using 30-fs-class lasers.

Table I shows parameters for different scenarios for strongly radiation-damped $\left(\psi_{Q} \gtrsim 1\right)$ and quantumelectrodynamically strong $\left(\chi_{e} \gtrsim 1\right)$ physics in a nonlinear Thomson or Compton scattering geometry for an 800-nm laser pulse with intensity $I_{\mathrm{L}}$ colliding with an electron beam with energy $E_{\mathrm{b}}$. Row (a) in Table I corresponds to the SLAC experiment [50]. Row (b) corresponds to nearterm experiments using intense $30-\mathrm{fs}$ lasers such as Hercules [45] or Astra Gemini [46] and a laser-wakefield-generated electron beam. Row (c) corresponds to an "ideal" experiment using two laser-beam lines (as Astra Gemini has access to) with the current maximum experimentally demonstrated laser intensity [53] and laser-wakefield-accelerated electron-beam energy [54-58]. The SLAC experiment is shown for comparative purposes only since the quasistatic field approximation is not valid for this case [59].

In case (a), the laser vector potential is of the order of both $a_{\text {rad }}$ and $a_{Q}$. Case (b) corresponds to a situation in which there will be strong radiation damping but quantum effects will be weak: $a_{Q}>a_{0}>a_{\text {rad. }}$. In case (c), the laser is sufficiently intense for both radiation damping and quantum recoil to be manifest: $a_{0}>a_{Q}>a_{\text {rad }}$.

TABLE I. Different scenarios for strong radiation-damping $\left(\psi_{Q} \gtrsim 1\right)$ and QED strong $\left(\chi_{e} \gtrsim 1\right)$ physics in a nonlinear Thomson scattering geometry for $800 \mathrm{~nm}$ wavelength laser pulses with intensity $I_{L}$ colliding with an electron beam with energy $E_{b}$.

\begin{tabular}{lcccccccc}
\hline \hline & $E_{\mathrm{b}} / \mathrm{GeV}$ & $I_{\mathrm{L}} / \mathrm{W} \mathrm{cm}^{-2}$ & $a_{0}$ & $a_{\mathrm{rad}}$ & $a_{Q}$ & $\chi_{e}$ & $\psi$ & $\psi_{Q}$ \\
\hline (a) & 46.6 & $1 \times 10^{18}$ & 0.5 & 2.5 & 1.2 & 0.43 & 0.045 & 0.018 \\
(b) & 0.2 & $5 \times 10^{21}$ & 50 & 46 & 420 & 0.12 & 1.2 & 0.74 \\
(c) & 1 & $2 \times 10^{22}$ & 100 & 21 & 84 & 1.2 & 23 & 3.7 \\
\hline \hline
\end{tabular}


Since our model is classical-that is, involves equations of motion only-it is restricted to the parameter range where $\chi^{2} \ll 1$ [49]. For the parameters described here, $\chi^{2}=0.014$, so the classical approach is reasonable. This reasoning also motivates the the description of this process as "nonlinear Thomson scattering" rather than as "Compton" scattering. We also calculate the electron spectrum after the interaction in the presence of radiation damping with and without the $g$ factor, showing that quantum modifications to radiation losses may be measurable.

\section{THE MODEL AND NUMERICAL METHODS}

The spectral intensity of radiation emitted by a number $N_{\mathrm{P}}$ of accelerating point charges can be expressed, in the far field, as [23]

$$
\frac{d^{2} I}{d \omega d \Omega}=\frac{\mu_{0} e^{2} c}{16 \pi^{3}} \omega^{2}\left|\int_{-\infty}^{\infty} \sum_{j=1}^{N_{\mathrm{p}}} \hat{\mathbf{s}} \times \beta_{j} e^{i \omega\left(t-\mathbf{n} \cdot \mathbf{r}_{j} / c\right)} d t\right|^{2},
$$

where the unit vector $\hat{\mathbf{s}}$ is in the direction of observation, at a distance far compared with the scale of the emission region. Because the integral is over a function with a rapidly oscillating exponent, it will in general not converge without a time step obeying $\Delta \tau \ll 1 / \omega$ if a straightforward numerical integration is attempted [60-62]. Since we are interested in $\gamma$-ray photons in excess of an $\mathrm{MeV}$ energy generated from a few fs interaction, the ratio of the necessary time step to the integration time scale is computationally unfeasibly large. Recently, methods for overcoming this limit by using interpolation techniques or consideration of photon formation length have been developed [30,63-66]. Here, we use the method that we previously developed [30]; see that paper for further details of the numerical algorithm. The resolution in the simulation is chosen so that the overlap of consecutive interpolations is sufficiently smooth to minimize high-frequency artifacts. See Ref. [66] for a discussion of such issues.

The particle trajectories are calculated in the presence of four-potentials: $A^{\mu}=\left\{A^{0}=\phi / c, A^{1}, A^{2}, A^{3}\right\}$, representative of a spatiotemporally Gaussian laser pulse with no interaction between electrons. The laser pulse propagated in the $+\hat{\mathbf{x}}_{3}$ direction with four-potential described by

$$
A^{\mu}=\mathfrak{R}\left[\left\{A_{0}\right\}^{\mu}\left(x^{\alpha}\right) e^{i \kappa_{\alpha} x^{\alpha}} f\left(\kappa_{\alpha} x^{\alpha} / \omega_{0} t_{\mathrm{L}}\right)\right],
$$

where $\left\{A_{0}\right\}^{\mu}\left(x^{\alpha}\right)$ is the spatial distribution of fourpotential; in this case, $\kappa_{\alpha}=\left\{\omega_{0}, 0,0, \omega_{0} / c\right\}$ is the laser four-wave vector, and $f\left(\kappa_{\alpha} x^{\alpha} / \omega_{0} t_{\mathrm{L}}\right)$ is a function of time describing the temporal envelope. The spatial-temporal distribution of a tightly focused pulse that satisfies the vacuum Maxwell's equations is in general very complicated, but it is easier to formulate in terms of potentials than fields. The formulation is easier because it is possible to have a purely transverse (to propagation) vector potential and satisfy the vacuum Maxwell's equations, which is not possible with fields. This can be done by considering the Lorentz-invariant Lorenz gauge condition $\partial_{\mu} A^{\mu}=0$. Using a slowly varying envelope approximation, and using a transverse vector potential linearly polarized in the $\hat{\mathbf{x}}_{1}$ direction with propagation in the $\hat{\mathbf{x}}_{3}$ direction, the gauge condition can be approximated as $\left\{A_{0}\right\}^{0}=-\left(i c / \omega_{0}\right) \partial\left\{A_{0}\right\}^{1} / \partial x_{1}$ [67]. Here, vector and scalar potentials with corrections to the basic Gaussian optics formulation are introduced up to order $\theta_{0}^{2}$, where $\theta_{0}=2 c / \omega_{0} w_{0}$ is the asymptotic divergence angle of a Gaussian laser beam with a waist of $w_{0}$. This procedure yields potentials

$$
\begin{aligned}
& \left\{A_{0}\right\}^{1}=\left[1+\frac{\theta_{0}^{2}}{2}\left(\frac{1-i \zeta}{1+\zeta^{2}}\right)\left(1-\left(\frac{1-\zeta^{2}}{2\left(1+\zeta^{2}\right)}\right) \rho^{2}\right)\right] \Psi_{0}, \\
& \left\{A_{0}\right\}^{0}=i \theta_{0} \xi_{1} e^{-i \tanh ^{-1} \zeta}\left[\left\{A_{0}\right\}^{1}-\theta_{0}^{2}\left(\frac{1-\zeta^{2}}{1+\zeta^{2}}\right) \Psi_{0}\right],
\end{aligned}
$$

and $\left\{A_{0}\right\}^{2}=\left\{A_{0}\right\}^{3}=0$, where

$$
\Psi_{0}=\frac{e^{-i \tanh ^{-1} \zeta-(1+i \zeta) \rho^{2}}}{\sqrt{1+\zeta^{2}}},
$$

$\rho=\sqrt{x_{1}^{2}+x_{2}^{2}} / w_{0}, \quad$ and $\zeta=x_{3} \theta_{0} / w_{0} . \quad w_{0} / \theta_{0}$ is the Rayleigh range of the laser. Higher-order corrections to the field structure could be employed to account for extremely tight focusing, but here we restrict our numerical calculations to foci with $w_{0}>\lambda$, where $\lambda$ is the laser wavelength. Although these corrections in $\theta_{0}^{2}$ are of magnitude $\left(1 / \pi^{2}\right) \lambda^{2} / w_{0}^{2}$, so they are up to $10 \%$ of the zero-order fields and cannot be considered negligible, the next-order corrections are $\theta_{0}^{4}$ and therefore of less importance.

An electron beam is modeled using $N_{\mathrm{P}}$ particles initiated with a momentum $p_{0}$ in the $-\hat{\mathbf{x}}_{3}$ direction in front of the laser. In order to simulate a more realistic beam, rejection sampling against a Gaussian probability-distribution function is used to generate a beam with a spread in momentum, $\sigma_{\mathbf{p}}$, and position, $\sigma_{\mathbf{x}}$ that statistically approximated the phase-space distribution,

$$
f_{e}(\mathbf{x}, \mathbf{p}, t)=\exp \left[-\frac{x^{2}}{2 \sigma_{x}^{2}}-\frac{p^{2}}{2 \sigma_{p}^{2}}\right]
$$

where $x^{2} / \sigma_{x}^{2}=x_{1}^{2} / \sigma_{x_{1}}^{2}+x_{2}^{2} / \sigma_{x_{2}}^{2}+x_{3}^{2} / \sigma_{x_{3}}^{2}$ and $p^{2} / \sigma_{p}^{2}=$ $p_{1}^{2} / \sigma_{p_{1}}^{2}+p_{2}^{2} / \sigma_{p_{2}}^{2}+\left(p_{3}-p_{0}\right)^{2} / \sigma_{p_{3}}^{2}$. The root-mean-square transverse and longitudinal geometric emittances of the bunch are therefore given by $\epsilon_{\perp}=\pi \sqrt{\sigma_{p_{1}}^{2} \sigma_{x_{1}}^{2}+\sigma_{p_{2}}^{2} \sigma_{x_{2}}^{2}}$ and $\epsilon_{\|}=\pi \sigma_{p_{3}} \sigma_{x_{3}}$, respectively. Although the particletracking routine could easily calculate a much larger bunch, because of the computational demands of the numerical spectrometer for a full angular sweep, the 
TABLE II. Parameters for the electron beam used in the numerical model and emittance in real units for reference. Row A: Finite-momentum spread case. Row B: Zero-momentum spread case.

\begin{tabular}{ccccccc}
\hline \hline & $\sigma_{x_{1}}, \sigma_{x_{2}}$ & $\sigma_{x_{3}}$ & $\sigma_{p_{1}}, \sigma_{p_{2}}$ & $\sigma_{p_{3}}$ & $\epsilon_{\perp}$ & $\epsilon_{\|}$ \\
\hline $\mathrm{A}$ & $3 c / \omega_{0}$ & $9 c / \omega_{0}$ & $m_{e} c$ & $10 m_{e} c$ & $0.38 \pi \mathrm{mmmrad}$ & $61 \mathrm{MeV}$ fs \\
$\mathrm{B}$ & $3 c / \omega_{0}$ & $9 c / \omega_{0}$ & 0 & 0 & 0 & 0 \\
\hline \hline
\end{tabular}

number of electrons in the bunch has been limited to $N_{\mathrm{P}}=500$. Radiation from individual electrons is summed incoherently.

A Gaussian temporal envelope is used in all cases: $f=$ $e^{-\left(\kappa_{\alpha} x^{\alpha} / \omega_{0} t_{\mathrm{L}}\right)^{2}}$. The pulse duration is $t_{\mathrm{L}}=65 \omega_{0}$, where $\omega_{0}$ is the laser angular frequency, which in the case of a typical $0.8-\mu \mathrm{m}$ laser is $2.36 \times 10^{15} \mathrm{~s}^{-1}$, yielding $t_{\mathrm{L}}=27.5 \mathrm{fs}$ at $1 / e^{2}$ radius, or $32 \mathrm{fs}$ full-width-at-half-maximum, of intensity. The electron-beam parameters are varied, with $p_{0}$ corresponding to a beam energy typically of $204 \mathrm{MeV}$ $\left(\gamma_{0}=400\right)$. The linearly polarized laser, with normalized vector potential of $a_{0}=50$ corresponding to a peak laser intensity of $5.3 \times 10^{21} \mathrm{~W} \mathrm{~cm}^{-2}$, is focused to a spot with waist $w_{0}=2.55 \mu \mathrm{m}$, or $w_{0}=20 c / \omega_{0}$. The electronbeam parameters, comparable to those routinely achieved in laser-wakefield-acceleration experiments, are summarized in Table II.

\section{NUMERICAL RESULTS}

In this section, we detail real-world numerical calculations of a backscattering experiment applicable to near-term experiments using current laser systems and laser-wakefield-accelerated electrons. By "real world," we mean that the calculation of the radiation spectrum includes the effect of a Gaussian-shaped bunch of electrons with normalized emittance (longitudinal and transverse) comparable to that produced in laser-wakefield acceleration interacting with a tightly focused laser, that radiation-reaction forces are included, and that the radiation spectrum is calculated directly from the electron trajectories. However, the self-consistent absorption of laser pulse photons is not included. The energy radiated by a $10^{9}$-electron beam is shown later to be $0.3 \mathrm{~J}$, which is a non-negligible $2 \%$ of the pulse energy of the laser considered here. Including the depletion of laser energy would modify the spectrum of photons slightly but that is likely to be less important than the other effects we consider here.

The spectral intensity $d^{2} I / d \omega d \Omega$, where differential solid angle $d \Omega=\sin \theta d \theta d \phi$, is calculated on a grid consisting of 150 cells in $\omega$ over the range $10^{4} \omega_{0}<\omega<$ $10^{8} \omega_{0}$, with $\Delta \omega$ exponentially increasing with $\omega, 117$ cells in $\theta$ over the range $0<\theta<30 \mathrm{mrad}$ and 26 cells in $\phi$ over the range $0<\phi<\pi / 2 \mathrm{rad}$. For clarity in the figures, symmetry is assumed, and therefore the full ranges $-30<$ $\theta<30 \mathrm{mrad}$ and $0<\phi<\pi$ are displayed.

\section{A. The high-brilliance synchrotron source}

The properties of radiation from backscattering of an electron with a laser pulse have been extensively studied. We can therefore use analytic formulae to predict that, in the interaction of a electron beam of $\gamma=400$ with a laser of field strength $a_{0}=50$, the synchrotronlike spectrum will peak in energy at $\hbar \omega_{\text {peak }}=2.56 a_{0} \gamma^{2} \hbar \omega_{0}=30 \mathrm{MeV}$ [25]. Using the numerical model, we can more accurately model the properties of the radiation produced in a high-intensity laser interaction with a laser-wakefieldaccelerated electron beam as a source for applications. In particular, here we include the effects of radiation damping [20] and nonplane-wave laser fields [38] and calculate the full angular distribution of radiation.

Figure 3 shows the spectral intensity of radiation produced by a 500-electron bunch with $\gamma=400$ scattering from a laser pulse with $a_{0}=50$. In this example, the higher-order field contributions are included, as in Eqs. (16)-(18), as well as beam emittance as given in Table II A. These images represent reasonably realistic modeling of an experiment and results in a wellcollimated, smooth, synchrotronlike radiation emission extending up to very high energies, with a broad peak at approximately $10 \mathrm{MeV}$, which is a factor of 3 smaller than the analytic prediction due to the radiation-reaction and finite spot effects. Because the laser pulse is linearly polarized, as expected, the radiation is strongly polarized, but also the angular intensity distribution has a pronounced ellipticity, with the major axis in the direction of polarization. Linear polarization also leads to higher photon energies compared to a circularly polarized pulse with the same pulse energy.
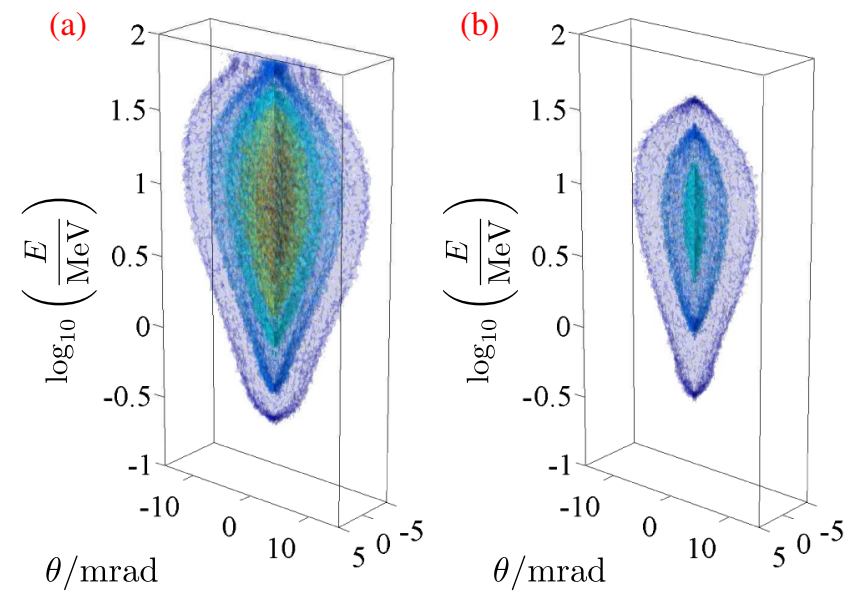

FIG. 3. The angularly resolved spectral intensity $\left(d^{2} I / d \omega d \Omega\right)$ due to a 500-electron bunch with $\gamma=400$ and emittance as given in Table II A, scattering from a laser pulse with $a_{0}=50$ with higher-order field contributions included as in Eqs. (16)-(18). The radiation-reaction force is not included in (a) and is included in (b). The contours are taken at identical spectral intensity levels for both cases, normalized to the peak, which is $1.6517 \times$ $10^{-26} \mathrm{Js}^{-1}$ at $0.2,0.3,0.4,0.5,0.6,0.7$, and 0.8 . 
One other notable effect is that of the higher-order terms in the laser fields. These do not significantly change the spectral shape, but they do change the magnitude nonnegligibly. Without the field contributions, the peak spectral intensity is $1.57 \times 10^{-26} \mathrm{Js}^{-1}$, but with them it is $1.65 \times 10^{-26} \mathrm{Js}^{-1}$, which is a $5 \%$ difference. Although the order $\theta^{2}$ pulse potential corrections have been simply added to the first order potential — so that the energy in the corrected pulse is higher than in the uncorrected-because the additional potential is approximately $10 \%$ of the firstorder potential, adding the corrections only represents about a $1 \%$ increase in pulse energy. The slight increase in pulse energy alone is too small to account for the increased radiation output. Instead, it is the additional longitudinal motion due to these potentials that increases the spectral output.

To compare this result to other synchrotron light sources, it is also useful to plot the on-axis spectrum in terms of the standard units of the synchrotron community: photons $\mathrm{s}^{-1} \mathrm{~mm}^{-2} \mathrm{mrad}^{-2} / 0.1 \%$ bandwidth. The spectral intensity is multiplied by a numerical factor that assumes that the 500 electrons are a reasonable statistical representation of a 100 -pC electron bunch that is typical of laser-wakefield experiments [2-4]. Also necessary for this calculation, the source size of the radiation is taken to be the laser spot area within the radius of half of the pulse waist, $\pi\left(w_{0} / 2\right)^{2}$. Although the source of the radiation is the electrons themselves, they are ponderomotively deflected during the interaction. We therefore use the laser-spot size rather than the electron-beam size as a more conservative estimate.

The on-axis radiation spectrum is shown in Fig. 4. As well as peaking at high energies, the peak spectral brilliance is also extremely high, comparable to the FLASH

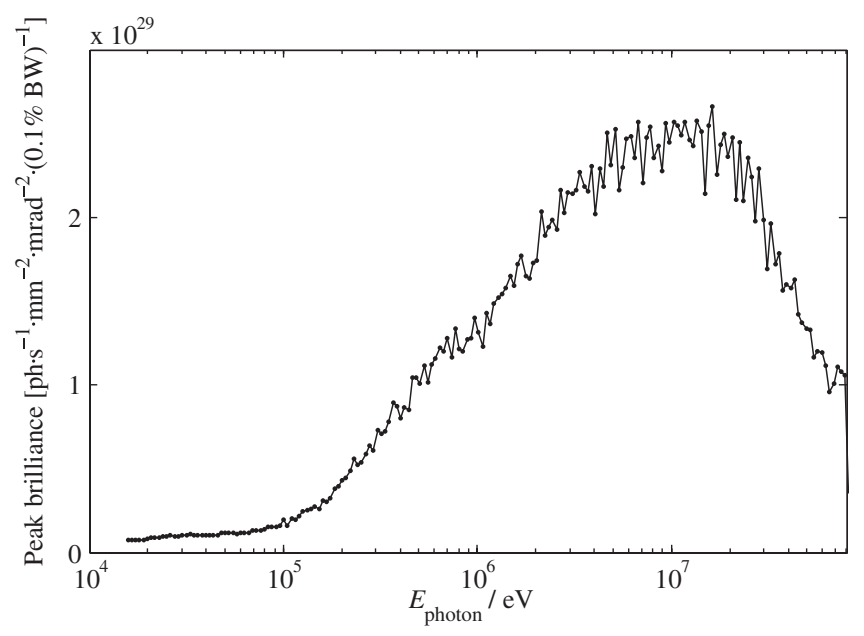

FIG. 4. The on-axis peak spectral brilliance, $d^{2} I / d \omega d \Omega /$ $\left(1000 \hbar t_{\mathrm{L}} \pi\left(w_{0} / 2\right)^{2}\right)$, in standard light-source units of photons $\cdot \mathrm{s}^{-1} \mathrm{~mm}^{-2} \mathrm{mrad}^{-2}(0.1 \% \text { bandwidth })^{-1}$ bandwidth due to a 100 -pC electron bunch with $\gamma=400$ and momentum spread given by Table II A scattering from a 30 -fs laser pulse with $a_{0}=50$.

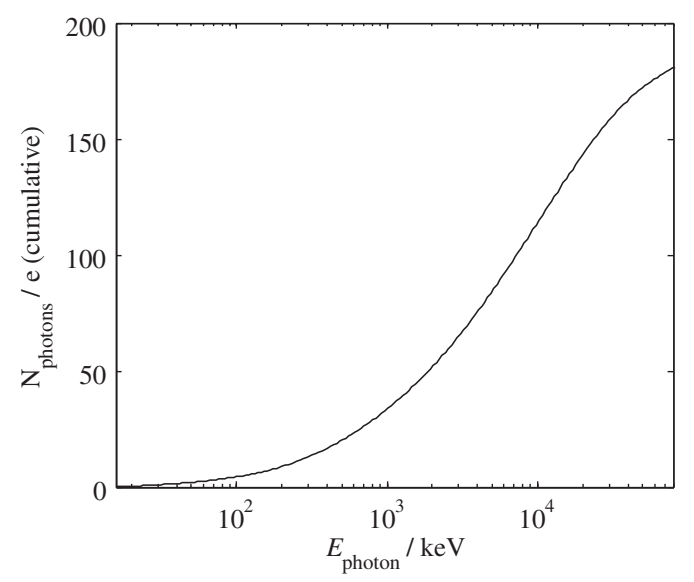

FIG. 5. The cumulative photon number $\left(\int_{0}^{E} d N / d E^{\prime} d E^{\prime}\right)$ per electron due to a 500-electron bunch with $\gamma=400$ and momentum spread scattering given by Table II A from a laser pulse with $a_{0}=50$.

free electron laser but at significantly higher photon energies [68] and significantly more brilliant than conventional synchrotrons. The effect of the high-intensity dramatically increases the brilliance of the source, at the expense of the bandwidth which at lower intensity can be extremely narrow, which may be of more utility for some applications [26-28].

In Fig. 5 the cumulative photon number, $\int_{0}^{E}\left(d N / d E^{\prime}\right) \times$ $d E^{\prime}$, per electron is shown for this spectrum, showing that on average each electron interacting with the laser field emits approximately 200 photons. When integrated numerically, the total photon energy emitted by each electron is $3.5 \times 10^{-10} \mathrm{~J}$. This is 10 times more than the energy of a $200-\mathrm{MeV}$ electron. This result may superficially appear not to conserve energy; however, the radiated photon energy is predominantly drawn from the laser pulse. For a bunch of $10^{9}$ electrons, which is of the order $100 \mathrm{pC}$ of charge, the total energy output would be $0.35 \mathrm{~J}$. For a bunch of this size, depletion of the laser fields - if treated self-consistentlywould modify the electron dynamics and radiation output, but should only be a small perturbation (the pulse energy used here is $19.0 \mathrm{~J}$ ) and hence would not be expected to modify this output energy significantly. Ignoring this correction, the conversion efficiency of laser-pulse energy into $\gamma$ rays is $1.8 \%$.

\section{B. On the observation of radiation-reaction effects in the photon distribution}

It has been suggested that signatures of the radiationreaction forces may be observed in the photon distribution emitted in a counterpropagating experiment [20,37]. The numerical calculations performed here suggest that this observation may be difficult due to the momentum spread of the electron beam. However, it should be noted that Di Piazza et al. [37] considered significantly larger angles of emission with relatively larger $a_{0}$ and smaller $\gamma_{0}$, which 

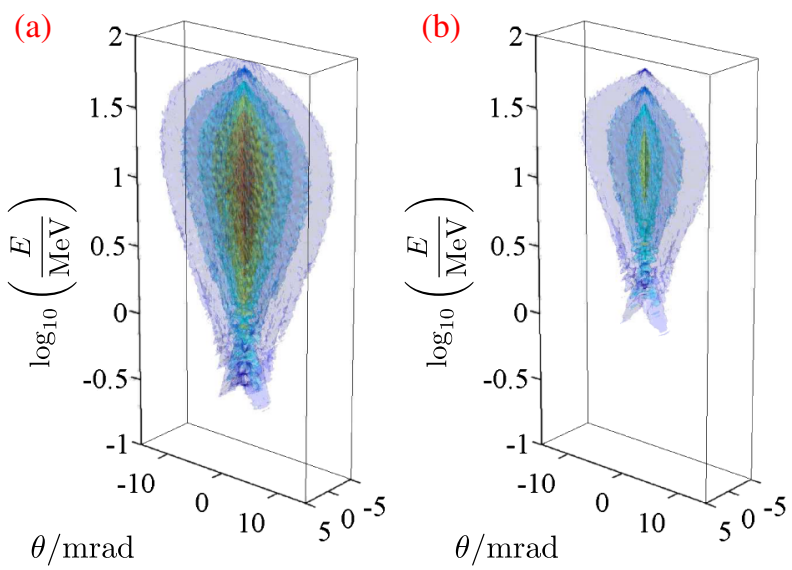

FIG. 6. The angularly resolved spectral intensity $\left(d^{2} I / d \omega d \Omega\right)$ due to a zero-emittance (Table II B) 500-electron bunch with $\gamma=400$ scattering from a laser pulse with $a_{0}=50$. The radiation-reaction force is not included in (a) and is included in (b). The contours are taken at identical spectral intensity levels for both cases, normalized to the peak, which is $2.6872 \times$ $10^{-26} \mathrm{Js}^{-1}$ at $0.2,0.3,0.4,0.5,0.6,0.7$, and 0.8 .

allowed changes to the radiation spectrum to be observable. Figure 6 shows the spectral intensity of radiation emitted under conditions identical to those of Fig. 3 except that here the electron beam has zero momentum spread, as in Table II B. The distribution has fine features that are smoothed out when the electron beam has a momentum spread, as would be expected. To see more clearly the effect of momentum spread on the radiation distribution, Figs. 7 and 8 show two-dimensional slices through the radiation intensity distribution, in the planes parallel and perpendicular to the laser polarization. In addition, the spectral intensity has been converted into a photon distribution per electron, $\omega_{0} d^{2} N / d \omega d \Omega$, which is more likely to be the form of data obtained in an experiment (i.e., a histogram of photon hits on an array of single-photon counting detectors).

Figure 7 shows the photon distribution from a zero-momentum-spread electron-beam interaction. In (a) and (c), radiation-reaction force is not included, and in (b) and (d), radiation-reaction force is included. (a) and (b) show the photon distribution in the plane perpendicular to the laser polarization. (c) and (d) show the photon distribution in the plane parallel to the laser polarization. The angular distribution of photons shows pronounced differences with and without radiation-reaction forces, and the energy distribution is also dramatically changed, in particular resulting in a large number of low-energy photons in the damped case compared to no damping. Another feature is slow oscillations in the spectral intensity with frequency and energy. These oscillations may be due to the short truncated electron bunch and laser pulse in the time domain, which result in long wavelength oscillations in the frequency domain.

When the electron bunch is given the momentum spread of Table II A, the distinction between the cases with and without radiation-reaction force becomes significantly less. Figure 8 shows the photon distribution from this interaction. There is little difference in the spectral intensity distribution with and without radiation-reaction-force effects, except that the overall magnitude is reduced, and the peak energy is reduced. Differences in the angular distribution are small, however, and are likely to be much-smaller-than-expected shot-to-shot fluctuations in electron-beam emittance. Coupling this observation to the intrinsic difficulty of measuring high-energy photons in a collimated beam, it appears to be unfeasible that radiation-reaction effects will be discernible in experimental measurements in this configuration in the near term.

\section{On the observation of radiation-reaction effects in the electron phase-space distribution}

In contrast to the photon measurements, it should be very easy to observe radiation-reaction effects in the electrons as measured using a standard scintillatingscreen configuration. It is typical in laser-wakefieldaccelerator experiments to measure either the electron-beam profile using a scintillating screen, or

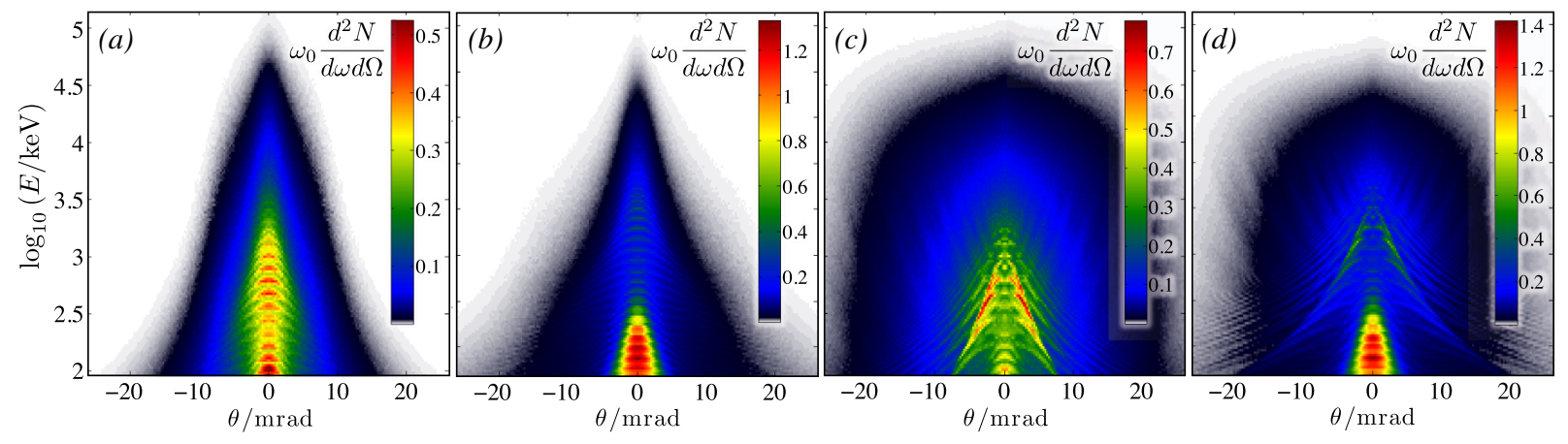

FIG. 7. The photon distribution (normalized to the laser frequency $\omega_{0} d^{2} N / d \omega d \Omega$ ) per electron due to a 500-electron bunch with $\gamma=400$ and zero momentum spread (Table II B) scattering from a laser pulse with $a_{0}=50$. In (a) and (c), radiation-reaction force is not included; in (b) and (d), radiation-reaction force is included. (a) and (b) show the photon distribution in the plane perpendicular to the laser polarization; (c) and (d) show the photon distribution in the plane parallel to the laser polarization. 

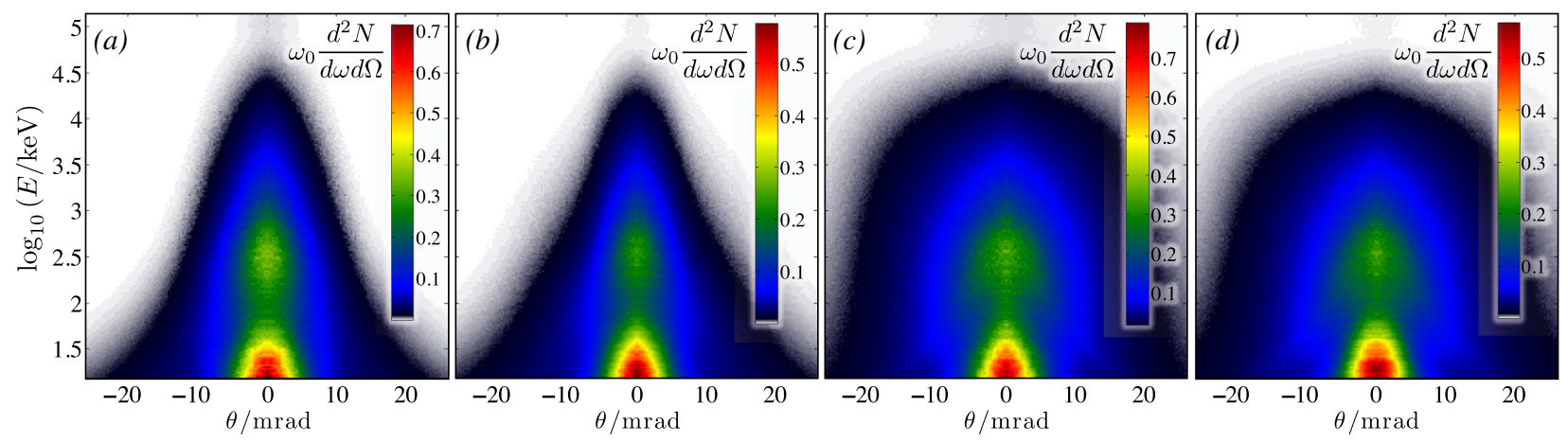

FIG. 8. The photon distribution (normalized to the laser frequency, $\omega_{0} d^{2} N / d \omega d \Omega$ ) per electron due to a 500-electron bunch with $\gamma=400$ and momentum spread given by Table II A scattering from a laser pulse with $a_{0}=50$. In (a) and (c), radiation-reaction force is not included; in (b) and (d), radiation-reaction force is included. (a) and (b) show the photon distribution in the plane perpendicular to the laser polarization; (c) and (d) show the photon distribution in the plane parallel to the laser polarization.

electron-forward-momentum spectrum using a deflecting magnet and a scintillating screen $[3,4]$. These diagnostics effectively correspond to the $p_{1}-p_{2}$ and $p_{1}-p_{3}$ electron phase-space densities, respectively-With a spectrometer, the deflection by the magnetic field disperses the electrons by $p_{3}$, but the projection in $p_{1}$ is maintained. In this section, only case A (i.e., including a momentum spread) is considered.

Figure 9 shows the $p_{1}-p_{2}$ phase-space density for the electron bunch before and after the interaction as twodimensional histogram plots. The electrons are deflected by the laser fields so that the transverse momentum spread is increased in both cases, consistent with a ponderomotive deflection. However, there is little difference between the cases with and without radiation-reaction forces because the radiation-damping effect reduces both transverse and longitudinal momenta proportionally. (To lowest order, the
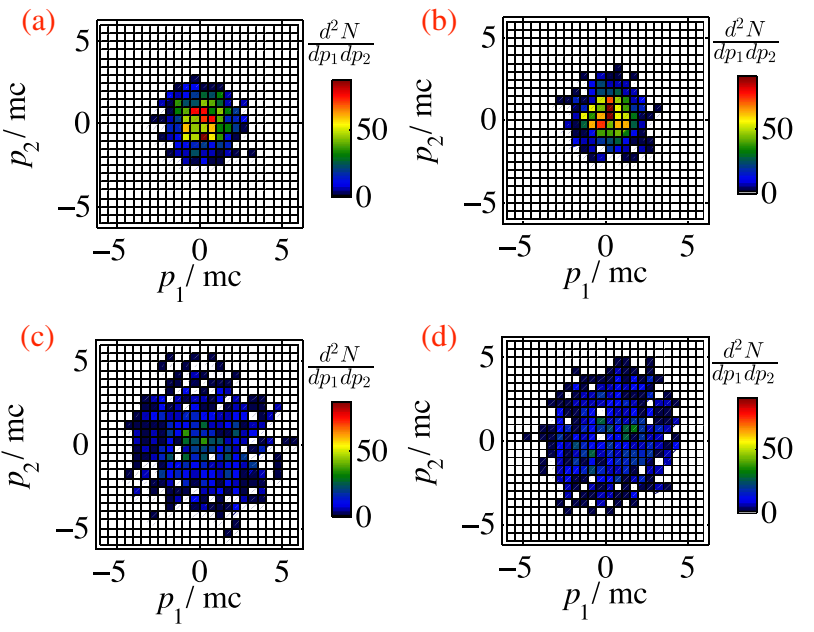

FIG. 9. Two-dimensional histograms of the $p_{1}-p_{2}$ phase-space distribution of a 500-electron bunch with momentum spread according to Table II A, before (top) and after (bottom) interaction with the high-intensity $\left(a_{0}=50\right)$ pulse. In (a) and (c), there is no radiation reaction; in (b) and (d), a radiation-reaction model is included according to Eq. (2). radiation force in Eq. (2) is $d p^{\mu} /\left.d \tau\right|_{\text {fric }}=-\tau_{0} \omega_{0}^{2} \gamma^{2} a^{2} p^{\mu}$.) Hence, in general, the exit angle of a particular electron $\theta_{\text {exit }} \simeq p_{\perp} / p_{3}$ is not expected to change significantly.

The effect on the electron spectrum is dramatic, however, as has also been previously shown by Koga et al. [20]. In Fig. 10, two-dimensional histogram plots of the $p_{3}-p_{1}$ phase-space density of the electron bunch are shown with and without radiation-reaction forces. Under the conditions modeled here, the electron beam loses almost half its energy when radiation damping is included (and as expected, it experiences little change in energy without radiation damping).

Finally, Fig. 11 shows two-dimensional histogram plots of the $p_{3}-p_{1}$ phase-space density of the electron bunch similar to Fig. 10, but this time the radiation
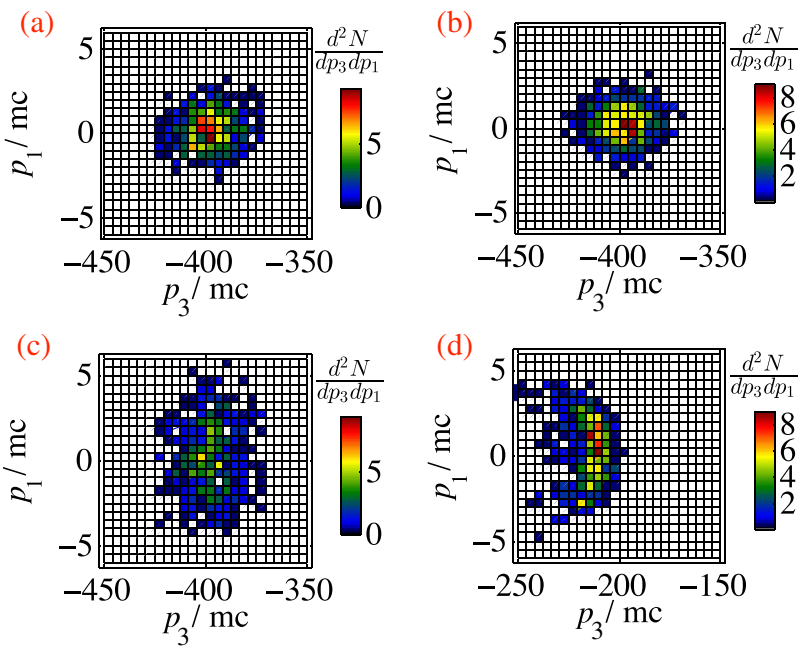

FIG. 10. Two-dimensional histograms of the $p_{3}-p_{1}$ phasespace distribution of a 500-electron bunch with momentum spread according to Table II A, before (top) and after (bottom) interaction with the high-intensity $\left(a_{0}=50\right)$ pulse. In (a) and (c), there is no radiation reaction; in (b) and (d), a radiationreaction model is included according to Eq. (2). Note that the horizontal momentum scale is negative and not the same for each phase space. 

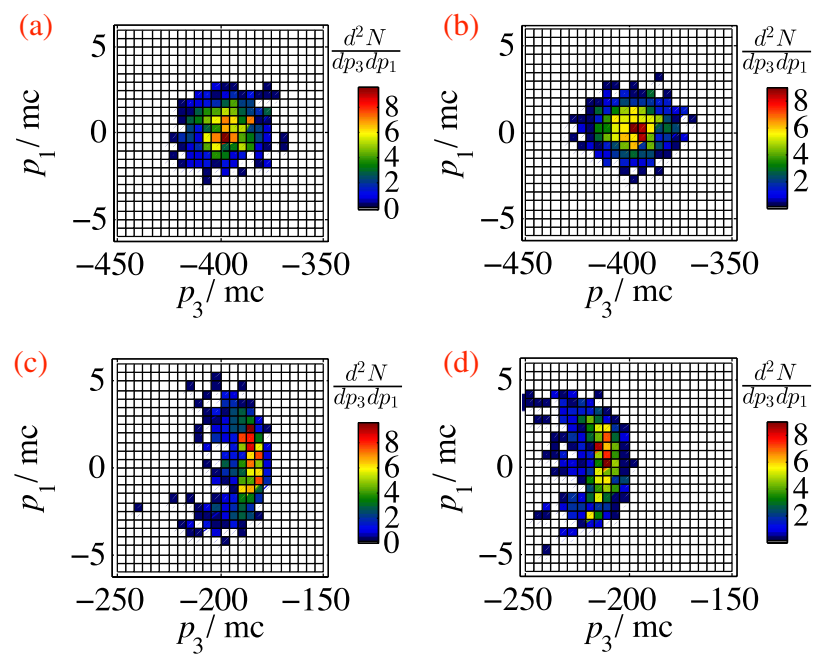

FIG. 11. Two-dimensional histograms of the $p_{3}-p_{1}$ phase-space distribution of a 500-electron bunch with momentum spread according to Table II A, before (top) and after (bottom) interaction with the high-intensity $\left(a_{0}=50\right)$ pulse with radiation damping. (a) and (c) use the purely classical expression of Eq. (2). In (b) and (d), the radiation-reaction four-force is modified by multiplying by the instantaneous $g$ factor given by Eq. (13). Note that the horizontal momentum scale is negative and not the same for each phase space.

reaction is shown with and without including the factor $g\left(\chi_{e}\right)$ given by Eq. (13). It can be seen by the plot that, under these conditions, the electron spectrum after the interaction with the $g$ factor differs from the purely classical result by about $10 \%$ relative to the overall energy loss. The smaller energy loss happens because the expected radiation spectrum is less energetic than the purely classical result would suggest. This difference between classical and quantum-corrected radiation reaction may be sufficiently large to be distinguishable over experimental fluctuations if well characterized. The effect of the addition of $g\left(\chi_{e}\right)$ on the photon spectrum calculated with classical radiation-reaction forces under these conditions is negligible.

\section{CONCLUSIONS}

The counterpropagating electron beam-ultra-highintensity laser interaction experiment described here is likely to be attempted by numerous groups in the near future. An experiment in the gamma-ray regime but with lower laser intensity has already recently been performed [22]. In addition to the ultimate study of quantumelectrodynamic effects, initial experiments with lower electron-beam energies and laser intensities are likely to be concerned with the brilliant high-energy photon output and classical forms of radiation forces. From these numerical calculations, we predict a large flux of photons with energy in excess of $1 \mathrm{MeV}$, in a beam collimated within a 10 -mrad divergence angle, and with an elliptical angular distribution due to the linear polarization of the laser pulse. Each electron should emit approximately 100 photons above $1 \mathrm{MeV}$ for a $200-\mathrm{MeV}$ Gaussian electron beam colliding with a pulse of intensity $5 \times 10^{21} \mathrm{~W} \mathrm{~cm}^{-2}$. For a typical laser-wakefield-accelerated electron bunch with $100-$ pC charge [2-4], this should result in about $10^{11}$ photons in a broad synchrotronlike spectrum peaking at $10 \mathrm{MeV}$ with approximately $2 \%$ conversion efficiency of laser energy into gamma rays, in a beam collimated to less than 10-mrad divergence and with a peak brightness exceeding $10^{29}$ photonss ${ }^{-1} \mathrm{~mm}^{-2} \mathrm{mrad}^{-2}(0.1 \% \text { bandwidth })^{-1}$.

In addition, we show that measurements of the radiation will unlikely be able to indicate signatures of radiationreaction forces, and, in particular, will unlikely have the ability to distinguish between different classical or quantum formulations of the radiation force, due to the effects of beam emittance and tight laser focusing. However, it should still be easy to observe radiation-reaction effects in the electron spectrum, where differences compared with a no-radiation force model are dramatic, even with moderate beam emittance. Including quantum effects using the $g\left(\chi_{e}\right)$ factor under these parameters causes a sufficiently reduced damping effect on the electron energy spectrum to be measurable.

Whether signatures of different classical radiationreaction force models can be observed in experiment is not addressed by the results of this paper. However, such observation is unlikely, since the primary measurable effect on the electrons is energy loss, which is to low order similar for all formulations of the radiation-reaction force. It is also likely that the differences between models will be hidden by the effects of beam emittance and laser focusing conditions.

\section{ACKNOWLEDGMENTS}

A. G. R. T. thanks A. Di Piazza and G. Sarri for useful discussions. This work was funded by the NSF under Contract Nos. 1054164 and 0935197, DARPA under Contract No. N66001-11-1-4208, EPSRC Grant Nos. EP/ I014462/1 and EP/G055165/1), and the Royal Society.

[1] T. Tajima and J. M. Dawson, Laser Electron Accelerator, Phys. Rev. Lett. 43, 267 (1979).

[2] S.P.D. Mangles, C. D. Murphy, Z. Najmudin, A. G. R. Thomas, J. L. Collier, A.E. Dangor, E. J. Divall, P. S. Foster, J. G. Gallacher, C. J. Hooker, D. A. Jaroszynski, A. J. Langley, W. B. Mori, P. A. Norreys, F. S. Tsung, R. Viskup, B. R. Walton, and K. Krushelnick, Monoenergetic Beams of Relativistic Electrons from Intense LaserPlasma Interactions, Nature (London) 431, 535 (2004).

[3] C. G. R. Geddes, Cs. Toth, J. van Tilborg, E. Esarey, C. B. Schroeder, D. Bruhwiler, C. Nieter, J. Cary, and W. P. Leemans, High-Quality Electron Beams from a Laser 
Wakefield Accelerator Using Plasma-Channel Guiding, Nature (London) 431, 538 (2004).

[4] J. Faure, Y. Glinec, A. Pukhov, S. Kiselev, S. Gordienko, E. Lefebvre, J.-P. Rousseau, F. Burgy, and V. Malka, A Laser-Plasma Accelerator Producing Monoenergetic Electron Beams, Nature (London) 431, 541 (2004).

[5] A. Rousse, K. T. Phuoc, R. Shah, A. Pukhov, E. Lefebvre, V. Malka, S. Kiselev, F. Burgy, J. P. Rousseau, D. Umstadter, and D. Hulin, Production of a keV X-Ray Beam from Synchrotron Radiation in Relativistic LaserPlasma Interaction, Phys. Rev. Lett. 93, 135005 (2004).

[6] Kim Ta Phuoc, Sebastien Corde, Rahul Shah, Felicie Albert, Romuald Fitour, Jean-Philippe Rousseau, Frederic Burgy, Brigitte Mercier, and Antoine Rousse, Imaging Electron Trajectories in a Laser-Wakefield Cavity Using Netatron X-Ray Radiation, Phys. Rev. Lett. 97, 225002 (2006).

[7] S. Kneip, S. R. Nagel, C. Bellei, N. Bourgeois, A.E. Dangor, A. Gopal, R. Heathcote, S. P. D. Mangles, J. R. Marques, A. Maksimchuk, P. M. Nilson, K. Ta Phuoc, S. Reed, M. Tzoufras, F. S. Tsung, L. Willingale, W. B. Mori, A. Rousse, K. Krushelnick, and Z. Najmudin, Observation of Synchrotron Radiation from Electrons Accelerated in a Petawatt-Laser-Generated Plasma Cavity, Phys. Rev. Lett. 100, 105006 (2008).

[8] H. P. Schlenvoigt, K. Haupt, A. Debus, F. Budde, O. Jäckel, S. Pfotenhauer, H. Schwoerer, E. Rohwer, J. G. Gallacher, E. Brunetti, R. P. Shanks, S. M. Wiggins, and D. A. Jaroszynski, A Compact Synchrotron Radiation Source Driven by a Laser-Plasma Wakefield Accelerator, Nature Phys. 4, 130 (2007).

[9] A. G. R. Thomas and K. Krushelnick, Betatron X-Ray Generation from Electrons Accelerated in a Plasma Cavity in the Presence of Laser Fields, Phys. Plasmas 16, 103103 (2009).

[10] S. Kneip, C. McGuffey, J. L. Martins, S. F. Martins, C. Bellei, V. Chvykov, F. Dollar, R. Fonseca, C. Huntington, G. Kalintchenko, A. Maksimchuk, S.P. D. Mangles, T. Matsuoka, S. R. Nagel, C. A. J. Palmer, J. Schreiber, K. Ta Phuoc, A. G. R. Thomas, V. Yanovsky, L. O. Silva, K. Krushelnick, and Z. Najmudin, Bright Spatially Coherent Synchrotron X-Rays from a Table-Top Source, Nature Phys. 6, 980 (2010).

[11] The ponderomotive expulsion of electrons occurs as a result of their time-averaged motion in the laser field, which leads to an effective force directed away from regions of high intensity. B. Quesnel and P. Mora, Theory and Simulation of the Interaction of Ultraintense Laser Pulses with Electrons in Vacuum, Phys. Rev. E 58, 3719 (1998).

[12] E. Esarey, S. K. Ride, and P. Sprangle, Nonlinear Thomson Scattering of Intense Laser-Pulses from Beams and Plasmas, Phys. Rev. E 48, 3003 (1993).

[13] F. V. Hartemann and A. K. Kerman, Classical Theory of Nonlinear Compton Scattering, Phys. Rev. Lett. 76, 624 (1996).

[14] Y. I. Salamin and F. H. M. Faisal, Generation of Compton Harmonics by Scattering Linearly Polarized Light of Arbitrary Intensity from Free Electrons of Arbitrary Initial Velocity, J. Phys. A 31, 1319 (1998).
[15] Y. Ueshima, Y. Kishimoto, A. Sasaki, and T. Tajima, Laser Larmor X-Ray Radiation from Low-Z Matter, Laser Part. Beams 17, 45 (1999).

[16] H. K. Avetissian and G. F. Mkrtchian, Nonlinear Quantum Regime of the X-Ray Compton Laser, Phys. Rev. E 65, 046505 (2002).

[17] K. Lee, Y.H. Cha, M. S. Shin, B.H. Kim, and D. Kim, Temporal and Spatial Characterization of Harmonics Structures of Relativistic Nonlinear Thomson Scattering, Opt. Express 11, 309 (2003).

[18] W. J. Brown, S. G. Anderson, C. P. J. Barty, S. M. Betts, R. Booth, J. K. Crane, R. R. Cross, D. N. Fittinghoff, D. J. Gibson, F. V. Hartemann, E. P. Hartouni, J. Kuba, G. P. Le Sage, D. R. Slaughter, A. M. Tremaine, A. J. Wootton, P. T. Springer, and J.B. Rosenzweig, Experimental Characterization of an Ultrafast Thomson Scattering $X$ Ray Source with Three-Dimensional Time and FrequencyDomain Analysis, Phys. Rev. ST Accel. Beams 7, 060702 (2004).

[19] F. V. Hartemann, D. J. Gibson, and A. K. Kerman, Classical Theory of Compton Scattering: Assessing the Validity of the Dirac-Lorentz Equation, Phys. Rev. E 72, 026502 (2005).

[20] J. Koga, T.Z. Esirkepov, and S. V. Bulanov, Nonlinear Thomson Scattering in the Strong Radiation Damping Regime, Phys. Plasmas 12, 093106 (2005).

[21] F. Albert, S. G. Anderson, D. J. Gibson, R. A. Marsh, C. W. Siders, C.P. J. Barty, and F. V. Hartemann, ThreeDimensional Theory of Weakly Nonlinear Compton Scattering, Phys. Plasmas 18, 013108 (2011).

[22] K. Ta Phuoc, S. Corde, C. Thaury, V. Malka, A. Tafzi, J. P. Goddet, R. C. Shah, S. Sebban, and A. Rousse, All-Optical Compton Gamma-Ray Source, Nature Photon. 6, 308 (2012).

[23] John David Jackson, Classical Electrodynamics (John Wiley and Sons, New York, 1999), 3rd ed.

[24] Fei He, Y. Y. Lau, Donald P. Umstadter, and Richard Kowalczyk, Backscattering of an Intense Laser Beam by an Electron, Phys. Rev. Lett. 90, 055002 (2003).

[25] Y. Y. Lau, F. He, D. P. Umstadter, and R. Kowalczyk, Nonlinear Thomson Scattering: A Tutorial, Phys. Plasmas 10, 2155 (2003).

[26] F. V. Hartemann, D. J. Gibson, W. J. Brown, A. Rousse, K. Ta Phuoc, V. Mallka, J. Faure, and A. Pukhov, Compton Scattering X-Ray Sources Driven by Laser Wakefield Acceleration, Phys. Rev. ST Accel. Beams 10, 011301 (2007).

[27] F. Albert, S. G. Anderson, D. J. Gibson, C. A. Hagmann, M. S. Johnson, M. Messerly, V. Semenov, M. Y. Shverdin, B. Rusnak, A. M. Tremaine, F. V. Hartemann, C. W. Siders, D. P. McNabb, and C.P. J. Barty, Characterization and Applications of a Tunable, Laser-Based, MeV-Class Compton-Scattering Gamma-Ray Source, Phys. Rev. ST Accel. Beams 13, 070704 (2010).

[28] F. Albert, S. G. Anderson, D. J. Gibson, R. A. Marsh, S. S. Wu, C. W. Siders, C. P. J. Barty, and F. V. Hartemann, Design of Narrow-Band Compton Scattering Sources for Nuclear Resonance Fluorescence, Phys. Rev. ST Accel. Beams 14, 050703 (2011).

[29] S. V. Bulanov, T.Zh. Esirkepov, Y. Hayashi, M. Kando, H. Kiriyama, J. K. Koga, K. Kondo, H. Kotaki, A. S. 
Pirozhkov, S.S. Bulanov, A. G. Zhidkov, P. Chen, D. Neely, Y. Kato, N. B. Narozhny, and G. Korn, On the Design of Experiments for the Study of Extreme Field Limits in the Interaction of Laser with Ultrarelativistic Electron Beam, Nucl. Instrum. Methods Phys. Res., Sect. A 660, 31 (2011).

[30] A. G. R. Thomas, Algorithm for Calculating Spectral Intensity Due to Charged Particles in Arbitrary Motion, Phys. Rev. ST Accel. Beams 13, 020702 (2010).

[31] P. A. M. Dirac, Classical Theory of Radiating Electrons, Proc. R. Soc. A 167, 148 (1938).

[32] F. Rohrlich, Dynamics of a Charged Particle, Phys. Rev. E 77, 046609 (2008).

[33] Igor V. Sokolov, Natalia M. Naumova, John A. Nees, Gerard A. Mourou, and Victor P. Yanovsky, Dynamics of Emitting Electrons in Strong Laser Fields, Phys. Plasmas 16, 093115 (2009).

[34] Richard T. Hammond, Radiation Reaction at Ultrahigh Intensities, Phys. Rev. A 81, 062104 (2010).

[35] L. D. Landau and E. M. Lifshitz, The Classical Theory of Fields (Pergamon, Oxford, 1975).

[36] Sergei V. Bulanov, Timur Zh. Esirkepov, Masaki Kando, James K. Koga, and Stepan S. Bulanov, Lorentz-AbrahamDirac versus Landau-Lifshitz Radiation Friction Force in the Ultrarelativistic Electron Interaction with Electromagnetic Wave (Exact Solutions), Phys. Rev. E 84, 056605 (2011).

[37] A. Di Piazza, K. Z. Hatsagortsyan, and C. H. Keitel, Strong Signatures of Radiation Reaction below the RadiationDominated Regime, Phys. Rev. Lett. 102, 254802 (2009).

[38] F. V. Hartemann, A. L. Troha, H. A. Baldis, A. Gupta, A. K. Kerman, E. C. Landahl, N. C. Luhmann, and J. R. Van Meter, High-Intensity Scattering Processes of Relativistic Electrons in Vacuum and Their Relevance to High-Energy Astrophysics, Astrophys. J. Suppl. Ser. 127, 347 (2000).

[39] K. Lee, S. Y. Chung, S. H. Park, Y. U. Jeong, and D. Kim, Effects of High-Order Fields of a Tightly Focused Laser Pulse on Relativistic Nonlinear Thomson Scattered Radiation by a Relativistic Electron, Europhys. Lett. 89, 64006 (2010).

[40] V. I. Ritus, Tr. Fiz. Inst. Akad. Nauk SSSR 111, 6 (1979).

[41] G. Breit and John A. Wheeler, Collision of Two Light Quanta, Phys. Rev. 46, 1087 (1934).

[42] A. R. Bell and John G. Kirk, Possibility of Prolific Pair Production with High-Power Lasers, Phys. Rev. Lett. 101, 200403 (2008).

[43] A. M. Fedotov, N. B. Narozhny, G. Mourou, and G. Korn, Limitations on the Attainable Intensity of High Power Lasers, Phys. Rev. Lett. 105, 080402 (2010).

[44] Stepan S. Bulanov, Timur Zh. Esirkepov, A. G. R. Thomas, James K. Koga, and Sergei V. Bulanov, Schwinger Limit Attainability with Extreme Power Lasers, Phys. Rev. Lett. 105, 220407 (2010).

[45] V. Yanovsky, V. Chvykov, G. Kalinchenko, P. Rousseau, T. Planchon, T. Matsuoka, A. Maksimchuk, J. Nees, G. Cheriaux, G. Mourou, and K. Krushelnick, Ultrahigh Intensity-300-TW Laser at $0.1 \mathrm{~Hz}$ Repetition Rate, Opt. Express 16, 2109 (2008).

[46] C. J. Hooker, J. L. Collier, O. Chekhlov, R. Clarke, E. Divall, K. Ertel, B. Fell, P. Foster, S. Hancock,
A. Langley, D. Neely, J. Smith, and B. Wyborn, The Astra Gemini Project-A Dual-Beam Petawatt Ti:Sapphire Laser System, J. Phys. IV (France) 133, 673 (2006).

[47] J. Schwinger, On Gauge Invariance and Vacuum Polarization, Phys. Rev. 82, 664 (1951).

[48] Igor V. Sokolov, Natalia M. Naumova, John A. Nees, and Gerard A. Mourou, Pair Creation in QED-Strong Pulsed Laser Fields Interacting with Electron Beams, Phys. Rev. Lett. 105, 195005 (2010).

[49] Igor V. Sokolov, John A. Nees, Victor P. Yanovsky, Natalia M. Naumova, and Gerard A. Mourou, Emission and Its Back-Reaction Accompanying Electron Motion in Relativistically Strong and QED-Strong Pulsed Laser Fields, Phys. Rev. E 81, 036412 (2010).

[50] D. L. Burke, R. C. Field, G. Horton-Smith, J. E. Spencer, D. Walz, S. C. Berridge, W. M. Bugg, K. Shmakov, A. W. Weidemann, C. Bula, K. T. McDonald, E. J. Prebys, C. Bamber, S. J. Boege, T. Koffas, T. Kotseroglou, A. C. Melissinos, D. D. Meyerhofer, D. A. Reis, and W. Ragg, Positron Production in Multiphoton Light-by-Light Scattering, Phys. Rev. Lett. 79, 1626 (1997).

[51] Thomas Erber, High-Energy Electromagnetic Conversion Processes in Intense Magnetic Fields, Rev. Mod. Phys. 38, 626 (1966).

[52] J. G. Kirk, A. R. Bell, and I. Arka, Pair Production in Counter-Propagating Laser Beams, Plasma Phys. Controlled Fusion 51, 085008 (2009).

[53] S.-W. Bahk, P. Rousseau, T. A. Planchon, V. Chvykov, G. Kalintchenko, A. Maksimchuk, G. A. Mourou, and V. Yanovsky, Generation and Characterization of the Highest Laser Intensities $\left(10^{22} \mathrm{~W} / \mathrm{cm}^{2}\right)$, Opt. Lett. 29 , 2837 (2004).

[54] W. P. Leemans, B. Nagler, A. J. Gonsalves, C. S. Toth, K. Nakamura, C. G. R. Geddes, E. Esarey, C. B. Schroeder, and S.M. Hooker, GeV Electron Beams from a Centimetre-Scale Accelerator, Nature Phys. 2, 696 (2006).

[55] Nasr A. M. Hafz, Tae Moon Jeong, Il Woo Choi, Seong $\mathrm{Ku}$ Lee, Ki Hong Pae, Victor V. Kulagin, Jae Hee Sung, Tae Jun Yu, Kyung-Han Hong, Tomonao Hosokai, John R. Cary, Do-Kyeong Ko, and Jongmin Lee, Stable Generation of GeV-Class Electron Beams from Self-Guided Laser-Plasma Channels, Nat. Photonics 2, 571 (2008).

[56] S. Kneip, S. R. Nagel, S. F. Martins, S. P. D. Mangles, C. Bellei, O. Chekhlov, R. J. Clarke, N. Delerue, E. J. Divall, G. Doucas, K. Ertel, F. Fiuza, R. Fonseca, P. Foster, S. J. Hawkes, C. J. Hooker, K. Krushelnick, W. B. Mori, C. A. J. Palmer, K. Ta Phuoc, P. P. Rajeev, J. Schreiber, M. J. V. Streeter, D. Urner, J. Vieira, L. O. Silva, and Z. Najmudin, Near-GeV Acceleration of Electrons by a Nonlinear Plasma Wave Driven by a Self-Guided Laser Pulse, Phys. Rev. Lett. 103, 035002 (2009).

[57] C. E. Clayton, J. E. Ralph, F. Albert, R. A. Fonseca, S. H. Glenzer, C. Joshi, W. Lu, K. A. Marsh, S. F. Martins, W. B. Mori, A. Pak, F. S. Tsung, B. B. Pollock, J. S. Ross, L. O. Silva, and D.H. Froula, Self-Guided Laser Wakefield Acceleration beyond $1 \mathrm{GeV}$ Using Ionization-Induced Injection, Phys. Rev. Lett. 105, 105003 (2010).

[58] Haiyang Lu, Mingwei Liu, Wentao Wang, Cheng Wang, Jiansheng Liu, Aihua Deng, Jiancai Xu, Changquan Xia, Wentao Li, Hui Zhang, Xiaoming Lu, Cheng Wang, 
Jianzhou Wang, Xiaoyan Liang, Yuxin Leng, Baifei Shen, Kazuhisa Nakajima, Ruxin Li, and Zhizhan $\mathrm{Xu}$, Laser Wakefield Acceleration of Electron Beams beyond $1 \mathrm{GeV}$ from an Ablative Capillary Discharge Waveguide, Appl. Phys. Lett. 99, 091502 (2011).

[59] Huayu Hu, Carsten Müller, and Christoph H. Keitel, Complete QED Theory of Multiphoton Trident Pair Production in Strong Laser Fields, Phys. Rev. Lett. 105, 080401 (2010).

[60] L. N. G. Filon, On a Quadrature Formula for Trigonometric Integrals Proc. R. Soc. Edinburgh 49, 38 (1928).

[61] A. J. Jerri, Shannon Sampling Theorem-Its Various Extensions and Applications-Tutorial Review, Proc. IEEE 65, 1565 (1977).

[62] C.E. Shannon, Communication in the Presence of Noise, Proc. IRE 37, 10 (1949).

[63] J. L. Martins, S. F. Martins, R. A. Fonseca, and L. O. Silva, in Harnessing Relativistic Plasma Waves as Novel Radiation Sources from Terahertz to X-Rays and Beyond: Proceedings of SPIE-The International Society for Optical Engineering, Prague 2009, edited by D. A.
Jaroszynski and A. Rousse (SPIE Europe, Bellingham, Washington, 2009), Vol. 7359, p. 73590V.

[64] Lorenzo Sironi and Anatoly Spitkovsky, Synthetic Spectra from Particle-in-Cell Simulations of Relativistic Collisionless Shocks, Astrophys. J. Lett. 707, L92 (2009).

[65] J. L. Martins, S. F. Martins, R. A. Fonseca, and L. O. Silva, in Advanced Accelerator Concepts: 14th Workshop on Advanced Accelerator Concepts, Annapolis, MD, 2010, AIP Conference Proceedings Vol. 1299, edited by S.H. Gold and G.S. Nusinovich (American Institute of Physics, Melville, New York, 2010), pp. 191-196.

[66] Brian Reville and John G. Kirk, Computation of Synthetic Spectra from Simulations of Relativistic Shocks, Astrophys. J. 724, 1283 (2010).

[67] L. W. Davis, Theory of Electromagnetic Beams, Phys. Rev. A 19, 1177 (1979).

[68] Ian Robinson, Gerhard Gruebel, and Simon Mochrie, Focus on X-Ray Beams with High Coherence, New J. Phys. 12, 035002 (2010). 